\title{
Lessons from the recently halted microbicide trial in India
}

\author{
SUNEETA KRISHNAN'
}

'Visiting faculty, Centre for Public Policy, Indian Institute of Management, Bangalore; assistant adjunct professor, University of California, San Francisco, USA. Address for correspondence: Women's Global Health Imperative, 50 Beale Street, 12th floor, San Francisco, CA 94105, USA e-mail: suneeta.krishnan@gmail.com

In January 2007, two Phase III clinical trials of a candidate microbicide among women at high risk of HIV in India, South Africa, Benin, Uganda, and Nigeria were halted. This was done because analyses of preliminary data by the Independent Data Monitoring Committee indicated that the product was associated with an increased risk of acquiring HIV $(1,2)$. The planning, implementation, and subsequent cessation of these trials illustrate the mechanisms for protecting participants in microbicide trials in developing countries. The closure of the trials highlights the complexities of such decisions and the range of competencies that research teams must possess.

Microbicides are products that women can use to prevent sexually transmitted infections, including HIV. They can be produced in the form of gels, creams, or suppositories. Advocacy related to the development of microbicides is driven by the hope that these products will not only increase the number of HIV prevention options that are available to women, but also address the particular needs of women who are unable to negotiate the use of condoms with their male partners.

The microbicide being tested in the aforementioned trials was Ushercell, a gel containing cellulose sulfate (CS), a product that 11 prior safety and contraceptive trials in India, Africa, and the United States had indicated was safe for human use $(1,3)$. The CS trials were launched just a few years after a trial of Nonoxynol-9 (N-9), a spermicide that was hypothesised to be an effective microbicide, was stopped. The N-9 trial was halted when researchers observed an increased risk of HIV and concluded that the gel caused vaginal lesions that facilitated the acquisition of HIV (4).

Research on N-9 has contributed to a focus on evaluating the safety of microbicides. Indicators and markers of toxicity that are now routinely examined include signs and symptoms of genital irritation, changes in vaginal microflora, and the presence of inflammatory markers like cytokines. In the case of CS, there was no evidence of toxicity in the safety trials (3). A full analysis of the CS trial data, including an examination of other known factors that may explain the higher rate of seroconversion among CS users such as frequency of anal sex, condom use, and incidence of other sexually transmitted infections, will hopefully shed light on these unexpected results. The results also highlight that further research on indicators and predictors of harm is critical (5).

The standard of care offered to participants in clinical trials in developing countries, particularly by developed country sponsors, is a contentious issue. In the context of HIV prevention trials, this debate has centred on access to treatment for those who seroconvert during the trial (6). At a meeting convened by the WHO in July 2003, vaccine and microbicide researchers, ethicists and others reached a consensus that providing antiretroviral treatment (ART) to participants in HIV prevention trials was justified by the principles of beneficence (maximise benefits to participants), reciprocity (those who contribute important data to the study by becoming infected "deserve something in return"), and justice (all those who become infected should be treated equally). While few would now debate whether it is necessary to provide treatment, how it might be provided and who is responsible for providing treatment remain contentious.

The CS trial is among the first trials in which research organisations signed advance agreements with treatment providers (programmes run by the government and NGOs) and set aside funding for antiretroviral therapy and other care to participants who acquire HIV infection during the course of a trial (7). These agreements are at least in part a result of the considerable efforts of civil society organisations such as the Global Campaign for Microbicides (GCM) and the African Microbicides Advocacy Group. Their efforts have gone beyond ensuring such commitments to monitoring the realisation of these commitments. The GCM, for example, has independently monitored the provision of prevention and care services to trial participants by examining the trial's standard operating procedures and protocols, visiting sites, and interviewing study staff (5).

Among the safeguards that were established prior to the launch of the CS trial (and common in most clinical trials) are "stopping rules"-criteria and a timeline for assessing whether trial data indicate significant benefit or harm or are unlikely to yield a result (rendering the study futile). Data are monitored by a data safety monitoring board (DSMB) called the Independent Data Monitoring Committee in the CS trial. DSMBs consist of experts in statistics, medicine, and community issues, and these individuals are not directly involved in the trial. Formulating stopping rules and procedures for assessment is critical for both scientific and ethical reasons. An early stoppage of a trial may result in insufficient data to reach firm conclusions about the potential harm or benefit of a product, thereby jeopardising the interests of the participants (8). However, failure to stop a 
trial when harm is being caused is clearly an ethical violation.

Identifying stopping rules is a complex exercise, which requires weighing available scientific knowledge with ethical concerns. The ability to implement these rules is equally complex. The stoppage of the CS trial highlighted the importance of having a communication plan (9). These plans should include identifying and consistently interacting with various groups that are likely to be affected by the results of a trial, such as participating communities, media, advocacy groups, reporters, donors, and research partners; training researchers to interact with the media; and putting in place mechanisms for sharing information. A key lesson of the CS trial is that "communications capabilities [need to be] embedded in research" in order to ensure dissemination of accurate information (9).

The lessons we may draw for conducting clinical trials in India include the importance of the active involvement of civil society organisations to safeguard participants' rights, the need for careful balancing of ethical and scientific considerations when establishing DSMBs and trial stoppage rules, and attention to communication and dissemination of research. The engagement between the CS trial investigators and civil society organisations illustrates the opportunities and responsibilities that networks of individuals and organisations interested in bioethics in India can take on, as an increasing numbers of clinical trials are initiated in India. Expanding institutional ethics committees and DSMBs, and increasing the availability of training in research ethics all will be critical to ensure adequate protection of participants in Indian trials. Greater interaction between health advocates, researchers, media persons, and others is also needed to ensure effective communication of the risks and benefits of such research.

References

1. Press release. Phase III trials of cellulose sulfate microbicide for HIV prevention closed. Arlington, VA. 2007 Jan 31. [cited 2007 May 10]. Available from: http://www.conrad.org/press/phasehttp://www.conrad. org/pressroom.htmllltrials.htm

2. Gawrylewski A. Failure of HIV microbicide raises concerns. The Scientist [serial on the Internet]. 2007 Feb 21 [cited 2007 May 10]. Available from: http://www.the-scientist.com/news/display/52861/

3. Doncel G. Update on current microbicide trials: update on the CONRAD cellulosesulfate trial, preclinicalevaluation of cellulosesulfate. Presentation at the Fourteenth Conference on Retroviruses and Opportunistic Infections; 2007 Feb 25-28; Los Angeles. [cited 2007 May 12]. Available from: http://www.retroconference.org/2007/data/files/webpage_for_ CROI.htm

4. Van Damme L, Ramjee G, Alary M, Vyulsteke B, Chandeying V, Rees H, et al. Effectiveness of COL-1492, a nonoxynol-9 vaginal gel, on HIV-1 transmission in female sex workers: a randomised controlled trial. Lancet 2002; 360:971-7.

5. No author listed. Background information for advocates on cellulose sulfate trials. 2007 Feb 5. [cited 2007 May 12]. Available from: http:// www.global-campaign.org/CS-background.htm

6. Heise L, Wood S. Rethinking the ethical roadmap for clinical testing of microbicides. Washington, DC: Global Campaign for Microbicides; 2005. [cited 2007 May 12]. Available from: http://www.global-campaign.org/ researchethics.htm

7. No author listed. A joint statement issued by AMAG, GCM and AVAC - a civil society response. 2007 Jan 31. [cited 2007 May 12]. Available from: http://www.global-campaign.org/clientfiles/civil_soc_press_ release\%209.doc

8. Cannistra SA. The ethics of early stopping rules: who is protecting whom? J Clin Oncol 2004; 22: 1542-5. [cited 2007 May 14]. Available from http://jco.ascopubs.org/cgi/content/full/22/9/1542

9. Robinson ET, Shears KH. Embedding communication in microbicides research: Lessons from the cellulose sulfate trial closures. Presentation at the Alliance for Microbicide Development's annual meeting;2007 March 20-21; Washington, DC. [cited 2007 May 12]. Available from: http://www. microbicide.org/meetings/meeting10.shtml

\section{PUBLISHING DETAILS}

\section{Form IV (See Rule 8)}

1.Place of Publication : Forum for Medical Ethics Society, 0-18,"Bhavna”,Veer Savarkar Marg, Prabhadevi Mumbai 400025.

\section{Periodicity : Quarterly}

3. Printer's name : Dr Arun Bal

Nationality : Indian

Address : Flat 6, Mallika, Makrand Housing Society, VS Marg, Mahim, Mumbai 400016.

\section{Publisher's name : Dr Arun Bal}

Nationality : Indian

Address : Flat 6, Mallika, Makrand Housing Society, SVS Marg, Mahim, Mumbai 400016.

5. Editor's name : Dr George Thomas

Nationality : Indian

Address: 114J Rostrevor Garden, Teynampet, Chennai 600018

6. Name of Owner : Forum of Medical Ethics Society

Address : 0-18,'Bhavna', Veer Savarkar Marg, Prabhadevi, Mumbai 400025. 